Check for updates

Cite this: RSC Adv., 2019, 9, 491

\title{
Degradation of pendimethalin by the yeast YC2 and determination of its two main metabolites $\uparrow$
}

\author{
Yajie Han, (D) ${ }^{\text {ab }}$ Zonggui Tang, ${ }^{c}$ Huifang Bao, ${ }^{d}$ Dongmei Wu, ${ }^{e}$ Xiaolin Deng, ${ }^{f}$ \\ Gaowei Guo, ${ }^{f}$ Bang-Ce Ye iD *a and Bin Dai ${ }^{* a}$ \\ In this study, we isolated a yeast strain, YC2, by enrichment culture from pendimethalin-contaminated soil. The \\ analysis of its phenotypic features and 26S rRNA sequence confirmed that the strain YC2 is Clavispora lusitaniae. \\ According to the kinetics of pendimethalin degradation, $Y C 2$ could degrade $74 \%$ of $200 \mathrm{mg} \mathrm{L}^{-1}$ pendimethalin in \\ CMB liquid culture over 8 days. LC-MS/MS was used to identify the metabolites of pendimethalin after \\ degradation. This study confirmed that its metabolites consist of 1,2-dimethyl-3,5-dinitro-4- $N$ (buta-1,3-dien- \\ 2-yl)-dinitrobenzenamine- $N$-oxide and 1,2-dimethyl-3,5-dinitro-4- $N$ (prop-1-en-2-yl)-dinitrobenzenamine- $N$ - \\ oxide, which were broken down by a series of enzymatic reactions to produce $\mathrm{CO}_{2}$ and $\mathrm{H}_{2} \mathrm{O}$. Thus, the study \\ herein sheds light on the role of yeast in the degradation of pendimethalin.
}

Received 21st September 2018 Accepted 6th December 2018

DOI: $10.1039 / \mathrm{c} 8 \mathrm{ra0} 07872 \mathrm{f}$

rsc.li/rsc-advances remediation is an effective approach in the natural environment. $^{7,8}$ Degradation reactions by microorganisms include oxidation, reduction, hydrolysis, and dealkylation. Furthermore, bacteria and fungi are the main organisms for pesticide degradation. Fungi fine-tune the molecular structure of pesticides to make them non-toxic, which are further degraded under the action of soil bacteria. ${ }^{9}$ There are some reports on the degradation of pendimethalin by microbes of Azotobacter chroococcum, ${ }^{7}$ Bacillus sp. $\mathrm{Y},{ }^{10}$ Bacillus circulans, ${ }^{11}$ and fungi Aspergillus flavus. ${ }^{12}$ The latest study suggested that some fungi can degrade certain types of persistent or toxic environmental pollutants. Also, microbial communities can degrade pesticides from the environment. ${ }^{13,14}$ In previous reports, the microbial catabolism is initiated by different mechanisms, including nitroreduction, $N$-dealkylation, oxidation of benzene rings, and cyclization of side chains. ${ }^{15}$

In this study, we isolated a yeast strain, $\mathrm{YC} 2$, which is able to degrade pendimethalin. Strain YC2 initiated pendimethalin catabolism via the oxidation of the amine groups, generating two new metabolites, which were identified. Therefore, the catabolic pathway presented in this study is different from that in previous studies. Chemical Engineering of Xinjiang Bingtuan, Shihezi University, Shihezi 832003, People's Republic of China. E-mail: bcye@ecust.edu.cn; dbinly@126.com; Fax: +86993-2057270; Tel: +86993-2057270; +86993-2058176

${ }^{b}$ College of Life Science, Shihezi University, Shihezi 832003, People's Republic of China 'Analysis and Testing Center, Xinjiang Agricultural Reclamation Academy of Sciences, Shihezi 832000, People's Republic of China

${ }^{d}$ Institute of Applied Microbiology, Xinjiang Academy of Agricultural Sciences, Urumqi 830091, People's Republic of China

eBiotechnology Research Institue, Xinjiang Agricultural Reclamation Academy of Sciences, Shihezi, 832000, People's Republic of China

${ }^{f}$ Department of Chemistry, Teachers College, Shihezi University, Shihezi 832003, People's Republic of China

$\dagger$ Electronic supplementary information (ESI) available. See DOI: $10.1039 / \mathrm{c} 8 \mathrm{ra} 07872 \mathrm{f}$

\section{Materials and methods}

\subsection{Chemicals and reagents}

Pendimethalin EC 33\% was purchased from Jiangsu Fengshan Group, Corporation (in Jiangsu Province, China). The pendimethalin standard was purchased from Aladdin Bio-Chem Chemical Co. (Shanghai, China). All other chemicals were of analytical grade.

The YPD culture medium contained $20 \mathrm{~g} \mathrm{~L}^{-1}$ of peptone, $20 \mathrm{~g}$ $\mathrm{L}^{-1}$ of glucose and $10 \mathrm{~g} \mathrm{~L}^{-1}$ of yeast extract. Czapek Modified $\mathrm{A}$ 
(CMA) was used to find isolate yeasts, and the medium contained $2.0 \mathrm{~g} \mathrm{~L}^{-1} \mathrm{NaNO}_{3} ; 1.0 \mathrm{~g} \mathrm{~L}^{-1} \mathrm{~K}_{2} \mathrm{HPO}_{4} ; 0.5 \mathrm{~g} \mathrm{~L}^{-1} \mathrm{KCl} ; 0.5 \mathrm{~g}$ $\mathrm{L}^{-1} \mathrm{MgSO}_{4} \cdot 7 \mathrm{H}_{2} \mathrm{O}$; and $0.01 \mathrm{~g} \mathrm{~L}^{-1} \mathrm{FeSO}_{4} \cdot 7 \mathrm{H}_{2} \mathrm{O}$. To study the kinetics of pendimethalin degradation, Czapek Medium B (CMB) was used. The only difference is that Czapek medium A contains no sucrose and Czapek medium B contains $8 \mathrm{~g}$ sucrose per litre.

\subsection{Soil}

Yeast was collected from cotton cultivated soils in various areas in Shihezi (Xinjiang Province, China). These loamy soils were treated with pendimethalin annually for 15 years, and thus used for the isolation of the pendimethalin-degrading microbe. The soil samples were collected from the surface layer $(2-20 \mathrm{~cm})$. Selected samples were dried and sieved to $2 \mathrm{~mm}$ before the experiment.

\subsection{Enrichment and isolation of pendimethalin-degrading yeasts}

The pendimethalin-degrading yeasts were isolated using the enrichment culture technique. To isolate the yeasts, $10 \mathrm{~g}$ agricultural soil was added to a beaker containing $100 \mathrm{~mL}$ of CMA culture media supplemented with pendimethalin $\left(100 \mathrm{mg} \mathrm{L}^{-1}\right)$. The mixture in the beaker was incubated on a thermostatic shaker $(120$ $\mathrm{rpm}$ ) for 6 days at $30^{\circ} \mathrm{C}$ in the dark. Then $1 \mathrm{~mL}$ of suspension was removed and transferred to a flask containing fresh medium, and the solution was supplemented with pendimethalin $\left(200 \mathrm{mg} \mathrm{L}^{-1}\right)$ and incubated for another 6 days under the same conditions. Finally, the concentration of pendimethalin was adjusted in the range of 100 to $3000 \mathrm{mg} \mathrm{L}^{-1}$ at 6 days intervals over 2 months. The last enrichment medium was diluted in a gradient, and the dilutions were transferred to CMA agar-solidified medium with pendimethalin (100 $\mathrm{mg} \mathrm{L}^{-1}$ ), and then incubated at $30{ }^{\circ} \mathrm{C}^{16-19} \mathrm{Using}$ the streak plate technique, thirty strains were selected and stored at $-80{ }^{\circ} \mathrm{C}$ with $30 \%$ glycerol.

\subsection{Degradation of pendimethalin by the isolated strains}

To prepare the seed culture, the thirty strains were preincubated in YPD culture medium at $30{ }^{\circ} \mathrm{C}$ and shaken on a thermostatic shaker $(120 \mathrm{rpm})$ for $18 \mathrm{~h}$. Then $300 \mu \mathrm{L}$ seed culture was added to $10 \mathrm{~mL}$ of $\mathrm{CMB}$ supplemented with $200 \mathrm{mg} \mathrm{L}^{-1}$ pendimethalin (in a $50 \mathrm{~mL}$ flask). The CMB medium was put in the shaker $(120 \mathrm{rpm})$ at $30^{\circ} \mathrm{C}$. Then the cultures were centrifuged (9000 rpm, $10 \mathrm{~min}$ ) and the supernatant was extracted with petroleum ether $(10 \mathrm{~mL} \times 3)$. All the fractions were dried over anhydrous sodium sulphate and then distilled. Chromatographic grade methanol was utilized to dissolve the residue from the extraction. The concentration of pendimethalin was determined by HPLC using a C8 column $(5 \mu \mathrm{m}, 3.9 \times$ $150 \mathrm{~mm}$ ). The mobile phase was a mixture of chromatographic pure methanol and deionized water $(85: 15, \mathrm{v} / \mathrm{v})$ with a flow rate of $1 \mathrm{~mL} \mathrm{~min}-1$, and the peaks were measured at $238 \mathrm{~nm}$.

\subsection{Identification of strain YC2}

In the process of pendimethalin degradation, the best degrader of pendimethalin was selected and identified by $26 \mathrm{~S}$ rRNA gene sequence analysis with the primers NL1 (5'-GCA TAT CAA TAA GCG GAG GAA AAG- $3^{\prime}$ ) and NL4 (5'-GGT CCG TGT TTC AAG ACG G-3'). The NCBI BLASTN comparison software was used to reveal the taxonomic statue of the isolate. ${ }^{20}$ Furthermore, its microbiological properties were tested.

\subsection{Kinetics of pendimethalin degradation}

The strain YC2 was cultured in YPD medium and incubated at $30{ }^{\circ} \mathrm{C}$ for $18 \mathrm{~h}$ in a thermostatic chamber. The culture was centrifuged at $5000 \mathrm{rpm}$ for $10 \mathrm{~min}$ at $4{ }^{\circ} \mathrm{C}$, and the cell pellets were re-suspended with sterile distilled and then adjusted to 1.5 $\mathrm{OD}_{600}$. Afterwards, the cell suspension was used as the inoculum strain. The pendimethalin from CMA and CMB ( $200 \mathrm{mg} \mathrm{L}^{-1}$ ) was used as the production medium with a $2 \%$ size inoculums strain, and the samples were incubated in an incubator $(120 \mathrm{rpm})$ at $30{ }^{\circ} \mathrm{C}$ in the dark. The biodegradation of pendimethalin was evaluated at $24 \mathrm{~h}$ intervals. ${ }^{21-23}$ The $\mathrm{pH}$ value of the cultures was measured using a $\mathrm{pH}$ meter. The concentration of pendimethalin was determined by HPLC.

\subsection{Isolation and identification of metabolites}

HPLC-MS/MS was used to identify the components of the metabolites. Chromatographic separation of the metabolites was conducted on an UltiMate 3000 UHPLC system (Dionex, Thermo Fisher Scientific, USA) equipped with a Phenomenex synergy C18 column $(5.0 \mu \mathrm{m}, 250 \times 4.6 \mathrm{~mm})$ through gradient elution with the mobile phase consisting of $0.1 \%$ formic acidwater solution (A), and acetonitrile (B) at a flow rate of 1.0 $\mathrm{mL} \min ^{-1}$. The peak was measured at $233 \mathrm{~nm}$. The mass spectral analysis was performed using a Quadruple-Exactive Orbitrap mass spectrometer (Micromass, Thermo Fisher Scientific, USA), with a heat electrospray ionization probe (HESI) source. The used software for data collection was Xcalibur 2.2. Nitrogen was used as the sheath and auxiliary gas. The parameters of the instrument were as follows: sheath gas flow $50 \mathrm{arb}$, auxiliary gas flow $10 \mathrm{arb}$, source voltage $3.2 \mathrm{kV}$, capillary temperature $325{ }^{\circ} \mathrm{C}$, and heat temperature $350{ }^{\circ} \mathrm{C}$. The mass spectrometer was operated in Full MS/dd-MS ${ }^{2}$ experiment using the positive and negative modes, respectively. The full scan mode (from $\mathrm{m} / \mathrm{z} 100-1500$ ) was also used. The AGC target was 2 $\times 10^{5}$ and C-Trap max injection time was $80 \mathrm{~ms}$.

\section{Results and discussion}

\subsection{Isolation and identification of pendimethalin-degrading} strain YC2

Using an enrichment procedure, thirty strains that could degrade pendimethalin were screened; however, only one yeast strain, designated as YC2, showed the highest ability to degrade pendimethalin, which could degrade above $60 \%$ of $200 \mathrm{mg} \mathrm{L}^{-1}$ pendimethalin within 5 days. Thus, strain YC2 was selected for further study.

The strain YC2 colony grown on a YPD agar plate was nearly round, milky white and creamy, with a smooth surface, neat edges, viscous texture, buds and reproduction structures, and pseudohyphae. In addition, its microbiological, physiological 
Table 1 Results of the sugar fermentation and assimilation experiments

\begin{tabular}{|c|c|c|c|c|c|c|}
\hline Sugar experiment & Sucrose & Raffinose & D-Xylose & Lactose & Honey disaccharide & Glucose \\
\hline Fermentation & + & + & + & + & + & + \\
\hline Assimilation & + & + & + & + & + & + \\
\hline
\end{tabular}

Table 2 Results of the starch hydrolysis test, nitrogen assimilation experiment and litmus milk test

\begin{tabular}{llll}
\hline & \multicolumn{2}{l}{$\begin{array}{l}\text { Nitrogen assimilation } \\
\text { experiment }\end{array}$} & \\
\cline { 2 - 3 } Starch hydrolysis test & $\mathrm{KNO}_{3}$ & $\left(\mathrm{NH}_{4}\right)_{2} \mathrm{SO}_{4}$ & Litmus milk test \\
\hline- & - & + & - \\
\hline
\end{tabular}

and biochemical characters are shown in Tables 1, 2 and Fig. 1. Blast analysis of the 26S rRNA gene sequence of YC2 revealed a high sequence identity of up to $100 \%$ with Clavispora lusitaniae (Table 3). Thus, based on its phenotypic and phylogenetic properties, strain YC2 was identified as Clavispora lusitaniae.

\subsection{Kinetics of pendimethalin degradation}

The degradation and utilization of pendimethalin by Clavispora lusitaniae YC2 was identified on CMB culture, which was supplemented with $200 \mathrm{mg} \mathrm{L}^{-1}$ pendimethalin. The kinetics of pendimethalin degradation in the culture medium can be seen in Fig. 2. The result showed that strain YC2 could degrade $74 \%$ of $200 \mathrm{mg} \mathrm{L}^{-1}$ pendimethalin within 8 days of incubation, and Clavispora lusitaniae $\mathrm{YC} 2$ could efficiently degrade pendimethalin. Yeast can grow in the range of $\mathrm{pH}$ 3.0-7.5, and the optimum $\mathrm{pH}$ is 4.5 to 5.0 . We found that strain $\mathrm{YC} 2$ reduced the $\mathrm{pH}$ from

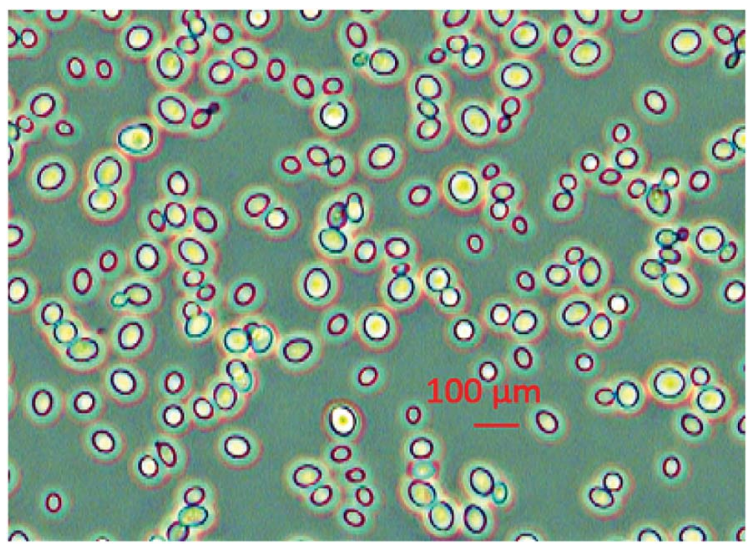

Fig. 1 Microbial morphology of YC2 under a microscope. the initial 7.51 to 5.5 during the degradation of pendimethalin, which is beneficial to the yeast growth and degradation activity.

\subsection{Identification of metabolites in liquid medium}

In liquid medium, according to HPLC and LC-MS/MS, there were two metabolites present during the degradation of pendimethalin. The ESI full scan is shown in Fig. 3, in which there are two peaks observed together with the parent peak (pendimethalin). The pendimethalin could be classified by comparing the reservation time of the standard $(10.2 \mathrm{~min})$. The other two peaks observed at $5.4 \mathrm{~min}$ and $6.2 \mathrm{~min}$ were denoted as $\mathrm{Y} 1$ and Y2, respectively.

Product Y1 was identified according to its mass spectrum data. As shown in Fig. 4B, its molecular ion appears at $\mathrm{m} / \mathrm{z} 267$ $\left([\mathrm{M}+\mathrm{H}]^{+}\right), m / z 289[\mathrm{M}+\mathrm{Na}]^{+}, m / z 533[2 \mathrm{M}+\mathrm{H}]^{+}$, and $m / z 555[2 \mathrm{M}$ $+\mathrm{Na}]^{+}$, fitting the molecular formula $\mathrm{C}_{13} \mathrm{H}_{12} \mathrm{~N}_{3} \mathrm{O}_{5}$. Based on the above results, metabolite $\mathrm{Y} 1$ is 1,2-dimethyl-3,5-dinitro-4$N$ (buta-1,3-dien-2-yl)-dinitrobenzenamine- $N$-oxide. Similarly, the mass spectrum of metabolite $\mathrm{Y} 2$ is shown in Fig. 4C, with its molecular ion at $m / z 279[\mathrm{M}+\mathrm{H}]^{+}, m / z 301[\mathrm{M}+\mathrm{Na}]^{+}$, and $m / z$ $579[2 \mathrm{M}+\mathrm{Na}]^{+}$, fitting the molecular formula of $\mathrm{C}_{11} \mathrm{H}_{12} \mathrm{~N}_{3} \mathrm{O}_{5}$. Thus, product $\mathrm{Y} 2$ was identified as 1,2-dimethyl-3,5-dinitro-4$N($ prop-1-en-2-yl)-dinitrobenzenamine- $N$-oxide.

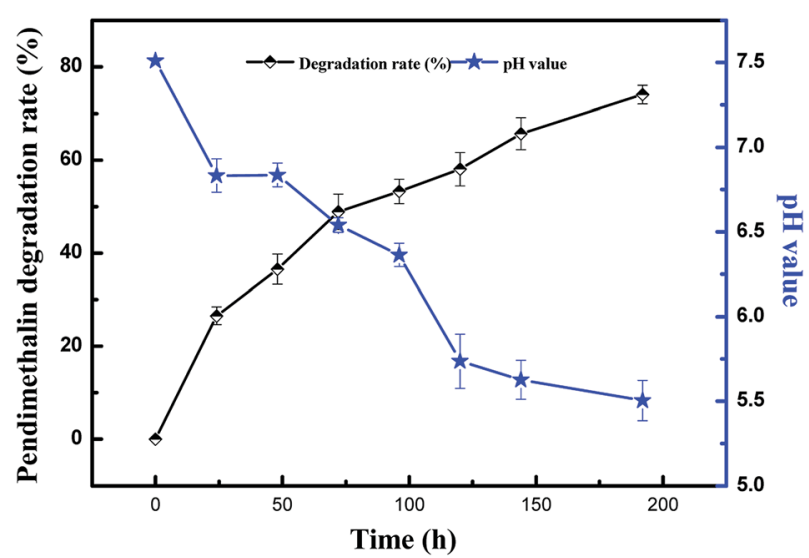

Fig. 2 Kinetics of the degradation of pendimethalin and variations in $\mathrm{pH}$ in the YC2 liquid culture. The results are representative of three experiments.

Table 3 Results of the blast for the YC2 26S rRNA gene sequence

\begin{tabular}{llll}
\hline Strain & Similar strain & Accession number & Ident. (\%) \\
\hline YC2 & Clavispora lusitaniae strain PMM10-1024036L & KP131848.1 & 100 \\
& Clavispora lusitaniae strain ATCC 34449 & KU729100.1 & 100 \\
& Clavispora lusitaniae & KY102567.1 & 100 \\
& Clavispora lusitaniae & MG599148.1 & 100
\end{tabular}



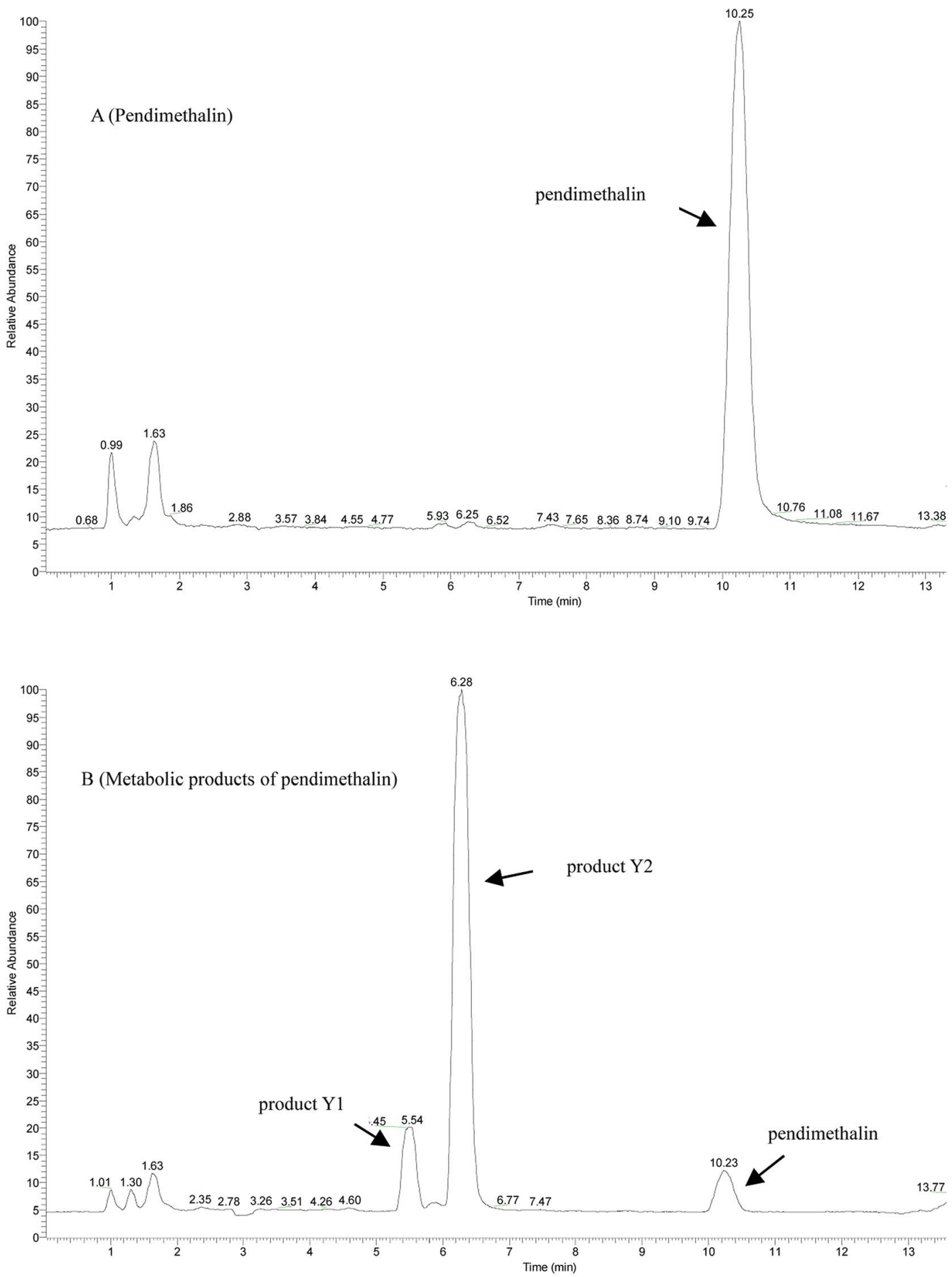

Fig. 3 ESI full scan of the metabolites degraded by YC2: (A) pendimethalin standard and (B) metabolites of pendimethalin.

\subsection{Metabolic pathway}

The presented metabolic pathway of pendimethalin in liquid medium is illustrated in Fig. 5. Since the liquid medium was an aerobic environment, the oxidation of the amine groups generated the product Y1 with the loss of $\mathrm{CH}_{4}$ and $\mathrm{H}_{2}$, and similarly the formation of metabolite $\mathrm{Y} 2$ with the loss of $\mathrm{C}_{2} \mathrm{H}_{6}$. Finally, these products were broken down by a subsequent series of enzymatic reactions to form carbon dioxide and water.
Previous reports have shown that some microorganisms can degrade pendimethalin, although the degradation pathways of pendimethalin are diverse. Two soil fungi, Fusarium oxysporum and Paecilomyces varioti degraded pendimethalin into two metabolites, that is $N$-(1-ethylpropyl)-3,4-dimethyl-2nitrobenzene-1,6-diamine and 3,4-dimethyl-2,6-dinitroaniline. ${ }^{24}$ Rhizoctonia bataticola resolved pendimethalin into 3,4dimethyl-2,6-dinitroaniline. Azotobacter 

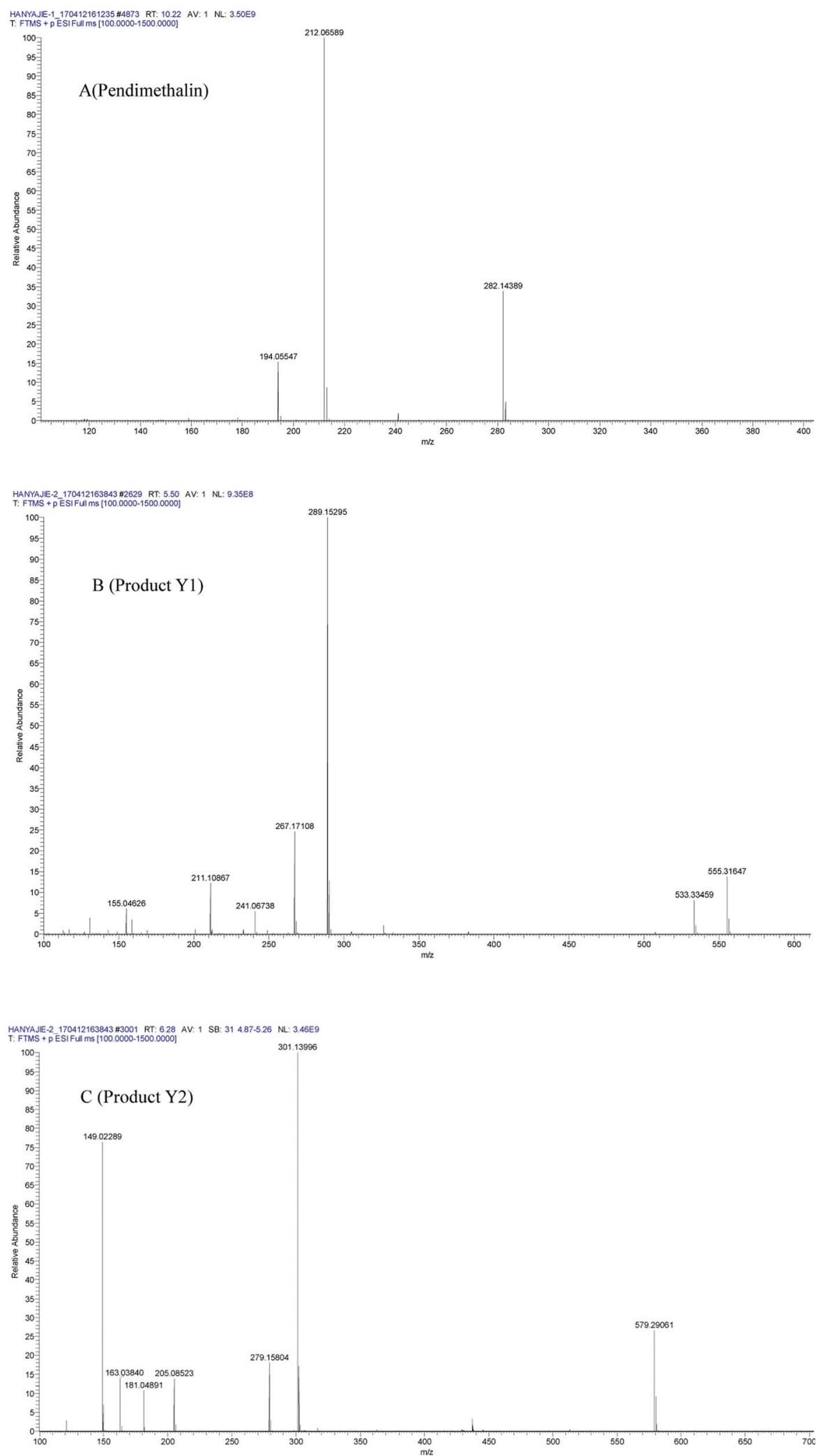

Fig. 4 MS/MS spectrum of the metabolic products of pendimethalin degraded by strain $\mathrm{Y} 3$. (A) Pendimethalin at RT and 10.22 min, (B) product $\mathrm{Y} 1$ at RT and $5.50 \mathrm{~min}$, and (C) product $\mathrm{Y} 2$ at RT and $6.28 \mathrm{~min}$.

decomposed pendimethalin into six metabolites: 3,4-dimethyl- methyl-2,4-dinitro-3-(pentan-3-ylamino)phenyl)methanol, and 2,6-dinitroaniline, 6-aminopendimethalin, $N$-(2,6-dinitro-3,4- 2-methyl-4-nitro-5- $N$-(1-cyclopropyl)-6-nitrosobenzyl alcohol. ${ }^{25}$ dimethyl) phenyl acetamide, 4,5-dimethyl-2-nitroaniline, (6- Pendimethalin was also degraded to 6-aminopendimethalin by 

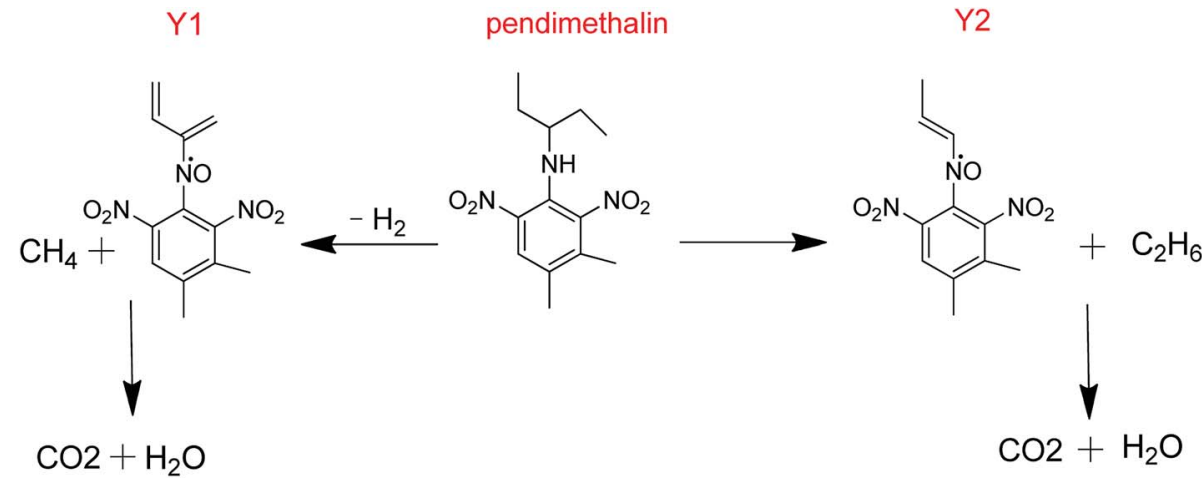

Fig. 5 Proposed metabolic pathway for pendimethalin by strain YC2.

nitroreduction, and other two downstream metabolites, 5amino-2-methyl-3-nitroso-4-(pentan-3-ylamino)benzoic acid and 8-amino-2-ethyl-5-(hydroxymethyl)-1,2-dihydroquinoxaline6-carboxylic acid were identified. ${ }^{26}$ Pendimethalin was also degraded to 1,3-dinitro-2-(pentan-3-ylamino)butane-1,4-diol by oxidative ring cleavage. ${ }^{7}$ Four metabolites were identified as $N$ (1-ethylpropyl)-3,4-dicarboxy-2,6-dinitrobenzenamine- $N$-oxide, $N$-(1-ethylpropyl)-3,4-dimethoxy-2,6-dinitrobenzenamine and benzimidazole-7-carboxyaldehyde in a bioslurry phase reactor. ${ }^{27}$

Therefore, our results are different from previous studies., ${ }^{520,28}$ The pathway in this study comes from the bioslurry reactor reported by Walker and Bond. ${ }^{27}$ Compared with other studies, the yeast in our study came from the natural soil, and we demonstrated that YC2 can be applied for the degradation of pendimethalin, which is new in the field of biodegradation mechanisms.

\section{Conclusions}

Strain YC2 was isolated from pendimethalin-contaminated soil and was characterized as Clavispora lusitaniae. Strain YC2 could degrade $74 \%$ of $200 \mathrm{mg} \mathrm{L}^{-1}$ pendimethalin within 8 days under incubation.

Two metabolites, 2-dimethyl-3,5-dinitro-4-N(buta-1,3-dien-2yl)-dinitrobenzenamine- $N$-oxide and 1,2-dimethyl-3,5-dinitro-4$N$ (prop-1-en-2-yl)-dinitrobenzenamine- $N$-oxide, were identified as the products of strain YC2-mediated degradation by LC-MS/ MS. Herein, we summarized the metabolic pathway of pendimethalin in liquid medium, and offered different results compared with previous studies. These findings may be a new degradation mechanism that differs from that in previous research.

\section{Conflicts of interest}

There are no conflicts to declare.

\section{Acknowledgements}

This study is supported by the National Natural Science Foundation of China (grant 21575089), Scientific Research Foundation for Changjiang Scholars of Shihezi University (CJXZ201501) and the Key Laboratory for Green Processing of Chemical Engineering of Xinjiang Bingtuan (No. KF201603).

\section{References}

$1 \mathrm{M}$. Younes and H. Galal-Gorchev, Pesticides in drinking water-a case study, Food Chem. Toxicol., 2000, 38(suppl. 1), S87-S90.

$2 \mathrm{~J}$. Lu, et al., Degradation of Pesticides in Nursery Recycling Pond Waters, J. Agric. Food Chem., 2006, 54(7), 2658-2663.

3 Y. D. Lee, et al., Loss of pendimethalin in runoff and leaching from turfgrass land under simulated rainfall, J. Agric. Food Chem., 2000, 48(11), 5376-5382.

$4 \mathrm{~L}$. Hou, et al., Pendimethalin exposure and cancer risk among pesticide applicators: a report from the U.S.-based agricultural health study, Ann. Epidemiol., 2004, 14(8), 608.

5 B. Singh and K. Singh, Microbial degradation of herbicides, Crit. Rev. Microbiol., 2016, 42(2), 245.

6 S. Venkata Mohan, M. Rama Krishna, et al., Solid phase bioremediation of pendimethalin in contaminated soil and evaluation of leaching potential, Bioresour. Technol., 2007, 98(15), 2905-2910.

7 R. K. Kole, et al., Bacterial degradation of the herbicide pendimethalin and activity evaluation of its metabolites, Bull. Environ. Contam. Toxicol., 1994, 52(5), 779.

8 C. Zhang, et al., Slurry-Phase Biological Treatment of 2,4Dinitrotoluene and 2,6-Dinitrotoluene: Role of Bioaugmentation and Effects of High Dinitrotoluene Concentrations, Environ. Sci. Technol., 2000, 34(13), 28102816.

9 M. C. Diez, Biological aspects involved in the degradation of organic pollutants, J. Soil Sci. Plant Nutr., 2010, 10(3), 244267.

$10 \mathrm{H}$. Ni, et al., Biodegradation of Pendimethalin by Paracoccus sp. P13, Curr. Microbiol., 2018, 75(8), 1077-1083.

11 V. B. Megadi, et al., Biodegradation of pendimethalin by Bacillus circulans, Indian J. Biotechnol., 2010, 9(2), 173-177.

12 A. S. Barua, et al., Degradation of pendimethalin by soil fungi, Pest Manage. Sci., 2010, 29(4), 419-425.

13 A. A. Juwarkar, et al., A comprehensive overview of elements in bioremediation, Rev. Environ. Sci. Bio/Technol., 2010, 9(3), 215-288.

14 A. P. Pinto, et al., Degradation of terbuthylazine, difenoconazole and pendimethalin pesticides by selected fungi cultures, Sci. Total Environ., 2012, 435-436(7), 402-410. 
15 R. K. Kole, et al., Bacterial degradation of the herbicide pendimethalin and activity evaluation of its metabolites, Bull. Environ. Contam. Toxicol., 1994, 52(5), 779-786.

16 N. K. Sahoo, et al., Biodegradation of 4-chlorophenol by Arthrobacter chlorophenolicus A6: effect of culture conditions and degradation kinetics, Biodegradation, 2011, 22(2), 275-286.

17 C. Li, et al., Biodegradation of methidathion by Serratia sp. in pure cultures using an orthogonal experiment design, and its application in detoxification of the insecticide on crops, Ann. Microbiol., 2013, 63(2), 451-459.

18 G. Chennappa, et al., Pesticide tolerant Azotobacter isolates from paddy growing areas of northern Karnataka, India, World J. Microbiol. Biotechnol., 2014, 30(1), 1-7.

19 A. P. Pinto, et al., Exploring the potential of novel biomixtures and Lentinula edodes fungus for the degradation of selected pesticides. Evaluation for use in biobed systems, Sci. Total Environ., 2016, 541, 1372-1381.

20 C. P. Kurtzman and C. J. Robnett, Identification of clinically important ascomycetous yeasts based on nucleotide divergence in the $5^{\prime}$ end of the large-subunit (26S) ribosomal DNA gene, J. Clin. Microbiol., 1997, 35(5), 1216.
21 Y. K. Kim, et al., Kinetics of endosulfan degradation by Phanerochaete chrysosporium, Biotechnol. Lett., 2001, 23(2), 163-166.

22 M. Goswami, et al., Kinetics of Chlorophenol Degradation by Benzoate-Induced Culture of Rhodococcus erythropolis M1, World J. Microbiol. Biotechnol., 2002, 18(8), 779-783.

23 S. S. Dash and S. N. Gummadi, Degradation Kinetics of Caffeine and Related Methylxanthines by Induced Cells of Pseudomonas sp, Curr. Microbiol., 2007, 55(1), 56-60.

24 S. B. Singh and G. Kulshrestha, Microbial degradation of pendimethalin, J. Environ. Sci. Health, Part B, 1991, 26(3), 309-321.

25 R. K. Kole, et al., Bacterial degradation of the herbicide pendimethalin and activity evaluation of its metabolites, Bull. Environ. Contam. Toxicol., 1994, 52(5), 779-786.

$26 \mathrm{H}$. Ni, et al., Biodegradation of pendimethalin by Bacillus subtilis Y3, J. Environ. Sci., 2016, 41(3), 121-127.

27 M. Ramakrishna, et al., Identification of metabolites during biodegradation of pendimethalin in bioslurry reactor, $J$. Hazard. Mater., 2008, 151(2-3), 658-661.

28 G. Kulshrestha and S. B. Singh, Influence of soil moisture and microbial activity on pendimethalin degradation, Bull. Environ. Contam. Toxicol., 1992, 48(2), 269-274. 